\title{
Employment of Telemedicine in Nursing Homes: Clinical Requirement Analysis, System Development and First Test Results
}

This article was published in the following Dove Press journal: Clinical Interventions in Aging

\author{
Marian Ohligs $\mathbb{D}^{1,2}$ \\ Stephanie Stocklassa' \\ Rolf Rossaint ${ }^{\prime}$ \\ Michael Czaplik (D) ${ }^{1,2}$ \\ Andreas Follmann (D) \\ 'Department of Anesthesiology, \\ University Hospital RWTH Aachen, \\ Aachen, NRW, Germany; ${ }^{2}$ Docs in \\ Clouds GmbH, Aachen, NRW, Germany
}

Correspondence: Marian Ohligs Department of Anesthesiology, University Hospital RWTH Aachen, Pauwelsstrasse 30, Aachen 52074, Germany

Tel +49-24I-8083I 36

$\mathrm{Fax}+49-24 \mathrm{I}-8082593$

Email mohligs@ukaachen.de
Purpose: Demographic change and lack of specialized workforces are challenging. Likewise, home visits by general practitioners (GPs) become rarer. If a nursing home resident develops acute symptoms, nurses are often inclined to call the rescue service. Besides patient-related consequences, this might lead to unnecessary hospitalization and farreaching health economic costs. Due to legal restrictions of remote treatment in Germany, which were recently loosened, telemedicine is still in the early stages. The aim of this study was to employ a holistic telemedical system for nursing homes which facilitates the connection to a GP and thus avoids unnecessary hospitalizations in the case of ambulatorysensitive illnesses.

Materials and Methods: After an inter-professional requirement analysis, the iterative development was started. In addition to an audio-video connection, several point of care measurements were integrated. Finally, first field tests were performed in a nursing home in a rural area in Germany.

Results: One nursing home was equipped with telemedical system based on the results of the requirement analysis and tele-medically connected to a GP. Over a period of seven months, 56 routine and emergency teleconsultations took place. Only one of those required a hospital admission. In addition to video telephony, electrocardiography and assessment of vitals such as pulse, blood pressure, oxygen saturation and auscultation of heart and lungs were applied frequently.

Conclusion: A telemedical system including integrated medical devices was successfully developed and has turned out to be helpful and even necessary for careful and reliable decision-making by the GP. First test results show high acceptance for elderly care. Involved patients, nurses, and the GP itemize various specific benefits, including economic, personal, and altruistic issues. Another issue that the current COVID-19 crisis brought to light is lowering the risk of contagion; GPs can replace their home visits by using telepresence combined with point of care measures.

Keywords: telemedicine, nursing homes, ban on visitors, COVID-19, teleconsultation, general practitioner care

\section{Introduction}

In many countries worldwide, the number of general practitioners (GPs) will decline in the coming years leading to a widening deficit in medical care, especially in rural areas. ${ }^{1}$ This trend is in serious contradiction to the rising demand for their services. By 2030, the number of older people will increase rapidly due to a demographic change. At the same time, the prevalence of chronic diseases is 
increasing. More than one in four adults suffers from chronical illness (often intertwined with complex comorbidities) and needs regular medical treatment. Moreover, younger people become increasingly affected by conditions such as type 2 diabetes and chronic cardiovascular diseases. ${ }^{2,3}$ Although GPs know their patients well and are able to detect even small changes in their patients' health states in an instant, they tend to suffer from high levels of routine stress due to the complex prevention and treatment requirements for chronically ill patients. ${ }^{4}$ The increasing demand for general medical care not only leads to an enormous financial burden for the health system but also generates a supply shortage, especially for the elderly population. ${ }^{5}$ Immobile patients living at home or in nursing homes are less frequently visited by GPs, if at all. Finding time for home visits presents a major administrative challenge for GPs because their waiting rooms are overcrowded. ${ }^{6-8}$ Accordingly, home visits are often linked to long waiting times.

At the same time, general medical care is crucial for elderly and chronically ill patients to prevent or alleviate exacerbations. Belated therapy is linked to hospitalization and often intensive care stays. ${ }^{9}$ Similar outcomes can be expected in the case of hypovolaemia, followed by exsiccosis, urinary tract infection, and sepsis. If acute symptoms occur, nurses are often inclined to call the rescue service (including an emergency physician) due to a lack of competence and authority. In many of these cases, relatively simple examination methods, such as the measurement of oxygen saturation, blood pressure, and pulse, or just the visual examination of an experienced physician would have already been sufficient for protecting the patient from distress due to unnecessary hospitalization and for saving on health care costs. ${ }^{10,11}$ Elderly people, especially suffering from dementia, tend to suffer from delirium when detached from their familiar environments. ${ }^{12}$

The teleconsultation is already firmly established within many areas of modern medicine. It ensures the spatially independent availability of medical expertise and offers often a diagnostic and treatment option for patients living in more rural areas. Accordingly, the physician's driving to the patients' nursing home can be omitted, which leads to a more efficient use of the personnel capacity. In many, although not in all situations, the teleconsultation allows the same diagnostic and treatment options as if the physician would be physically present. Even during prehospital emergencies, teleconsultations are suitable for initial diagnostic and treatment in these situations. Numerous studies have already confirmed that the quality of the supply has improved and that the scarce resource of a "physician" is being deployed purposefully. In addition, this has received great acceptance from all parties. $^{13-15}$

The advantages of telemedicine within in-house medical consultations are especially needed now due to the ever-increasing shortage of GPs. Here, specific clinical pictures and their follow-ups are discussed without going into detail on the general consultations or emergency situations of elderly patients. ${ }^{16}$ Further studies use only some of the aspects of telemedicine such as data management and storage without discussing the use of a single incorporated telemedical system. ${ }^{17}$ These systems can carry out various functions of vital data measurement and enable sufficient anamnesis via video communication. In this way, a diagnosis or a therapy, especially in cases of ambulatory care sensitive conditions, can be efficiently initiated by a GP. Unnecessary hospitalization can be avoided, patients can stay in their familiar environments, and the national health system can experience massive cost savings. ${ }^{18}$

In Germany, the development of telemedical applications was restrained due to legal restrictions of remote treatment of patients. Therefore, telemedicine is still in the early stages. Recently, those restrictions were loosened, so that nowadays medical therapies can be initiated without a previous personal contact. Certainly, these systems have to be certified in IT-Security, Data Privacy and their Content to fulfill the requirements of the "Federal Master Treaty for Medical Practitioners" (Bundesmantelvertrag für Ärzte) and the General Data Protection Regulation (GDPR) of the European Union.

The aim of this study was to develop a user-orientated, integrated telemedical system for pre-existing doctorpatient-relationships. Focus was placed on the feasibility of a structured video anamnesis and physical examination through telemedically connected devices in nursing homes. Focus was also placed on the evaluation of this system's acceptance by GPs and elderly patients.

\section{Materials and Methods}

A multi-layered development process was established for the development of a nursing care-specific telemedical system (Figure 1). Initially, an inter-professional requirement analysis was conducted. Subsequently, a prototype was developed and used for routine teleconsultations. After the initial onemonth introduction phase, a re-evaluation took place, leading 


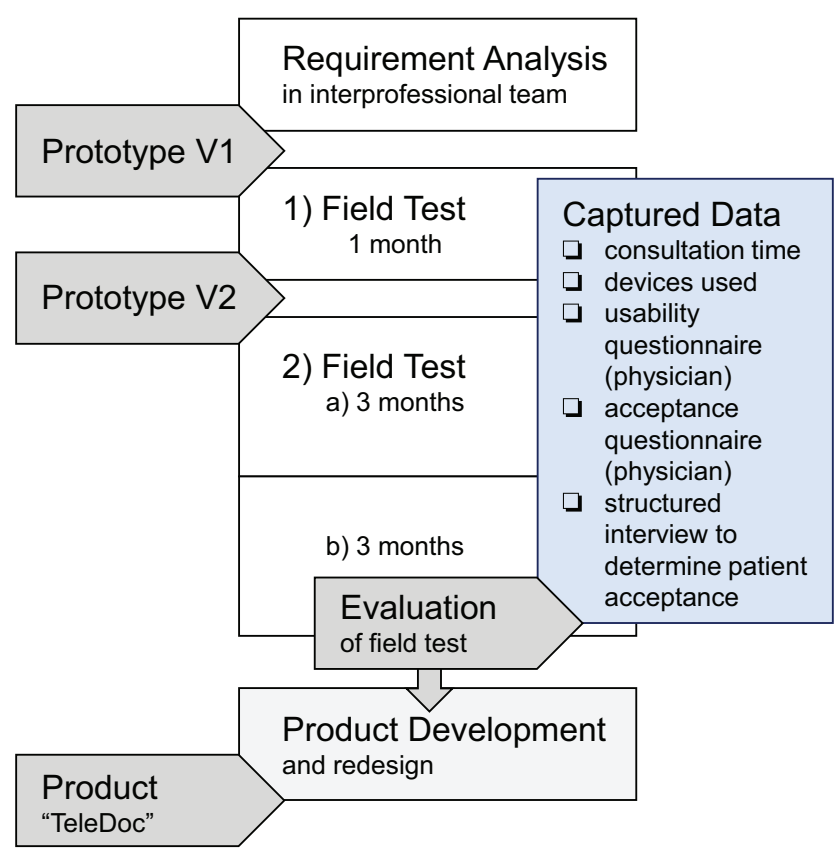

Figure I A multi-layered development process was established leading to three prototype versions. Field tests and user surveys contributed to the iterative improvements.

to further adaptations of the prototype. Another field test was executed over a period of six months. The results of all recorded parameters were subsequently analyzed and used for the industrial development of the so-called "TeleDoc" (Docs in Clouds TeleCare GmbH Aachen, Germany).

\section{Requirement Analysis and Development Process}

In the first stage of the developmental process, 24 major requirements (Table 1) were defined by an interdisciplinary team of involved nurses, a GP, and several elderly patients. Through the GP's experiences, specific needs could be integrated into the developed system. These include, in addition to good audio-video quality, the option of vital data measurement.

All groups agreed on the importance of complicationfree operations in both elective and emergency consultations. When the nurse called the GP, the telemedical system should be initiated both in the nursing home and GP practice. The GP could not only take the patient's medical history from the nurse or patient himself but also request the measurement of vital signs to convey therapy instructions. At the same time, the GP had the opportunity to assess the patient's physical appearance via video communication to build trust. All things considered, the telemedical system should facilitate an improved communication chain with a good working relationship among the GP, nurse, and patient (Figure 2).

Taking all formulated requirements into account, a telemedical communication, measurement and documentation system was developed and consecutively tested. Field testing in realistic conditions was enabled for the medicaltechnical assessment of the usability, durability, acceptance, and feasibility of the system, which was subsequently used for the iterative developmental process. After this initial testing, a further development led to an improved prototype.

\section{Field Tests}

After approval by the ethical commission (Aachen, Germany; EK 053/16), all participants provided informed consent, and which included, where applicable, publication of their images. Subsequently, the system was tested in a retirement home in a rural area (Wermelskirchen, Germany), linked to a particular GP practice. The retirement home is able to care for 99 elderly persons and has 52 supervised apartments. During the study 38 of the residents agreed to use teleconsultation. Twenty-eight persons used teleconsultations and 11 more than one time, in addition to home visits or normal consultations in the doctor's practice. The participants who agreed to state their name (18/28) aged between 61 and 99 (average: 83, median: 84). The GPs practice treats between 50 and 150 patients per day, by one doctor and four medical assistants. Between August 2017 and June 2018, the enhanced system was used for weekly routine rounds in the nursing home as well as for emergency consultations.

The testing period was divided into two phases. In the first phase between August and September 2017, the system (prototype 1) was used solely for routine consultations (Figure 1). A re-evaluation of requirements was subsequently executed, followed by a four-month adaption phase. Prototype version 2 was then used in a second field test between January 2018 and June 2018 for routine as well as emergency consultations. The routine consultations usually took place on Wednesdays. Shortly before the consultations, the nurses could discuss with the GP which patients needed to be viewed that day. Emergency consultations could take place at any time depending on the GP's availability. For evaluation, the second field test was divided into two timespans ( $2 \mathrm{a}$ and $2 \mathrm{~b}$ ).

\section{Data Assessment}

During the consultation, several factors were recorded for evaluation, including the duration of the consultation, the 
Table I List of Major Requirements Defined by the Interdisciplinary Team of Involved Nurses, a General Practitioner (GP) and Several Elderly Patients, Prioritized by Must, Should or May

\begin{tabular}{|c|c|c|c|}
\hline No & Brief Requirement Description & Priority & Source \\
\hline I & All data is transferred encrypted & MUST & GP, patient \\
\hline 2 & Doctor and nurse are able to see the important information of a consultation at anytime & MUST & GP \\
\hline 3 & Doctor and nurse are able to identify information lost during the transmission & MUST & GP, nurse \\
\hline 4 & Doctor and nurse are able to identify information distorted during the transmission & MUST & GP, nurse \\
\hline 5 & Doctor and nurse can resume the consultation if an error occurs & MUST & GP, nurse \\
\hline 6 & The video connection adapts compression, resolution and frame rate automatically in real-time & SHOULD & GP \\
\hline 7 & Patient can see and talk to the doctor & MUST & patient \\
\hline 8 & Nurse can see and talk to the doctor & MUST & GP, nurse \\
\hline 9 & Doctor and nurse can fill out the documentation together & MUST & GP, nurse \\
\hline 10 & $\begin{array}{l}\text { Medical devices for measurement of bloodpressure, oxygen saturation and electrocardiogram are part of the } \\
\text { system and automatically transfer the results to the general practitioner. }\end{array}$ & MUST & GP, nurse \\
\hline II & Doctor can see the patient & MUST & GP, nurse \\
\hline 12 & Doctor can see the patient and the nurse & MAY & GP \\
\hline 13 & Doctor can navigate and zoom the patient camera & SHOULD & GP \\
\hline 14 & The device has to be able to run as mobile device for 20 minutes without external power source & MUST & nurse \\
\hline 15 & The device is easily movable or portable between the residents' apartments & MUST & nurse \\
\hline 16 & The system can connect to the Internet via Wireless Local Area Network (WLAN) & MUST & nurse \\
\hline 17 & $\begin{array}{l}\text { The system can connect to the Internet via universal mobile telecommunication system (UMTS) or long-term } \\
\text { evolution (LTE) }\end{array}$ & MUST & nurse \\
\hline 18 & Login passwordless via smartcard & SHOULD & nurse \\
\hline 19 & All devices touched by the nurse are medically certified & MUST & GP, nurse \\
\hline 20 & All measurement units are certified medical devices & MUST & GP, nurse \\
\hline 21 & All components are Communauté Européenne (CE) certified products & MUST & GP, nurse \\
\hline 22 & Rolling stand pass the tilt-test for medical products in Germany & MUST & GP, nurse \\
\hline 23 & The documentation device has a keyboard & SHOULD & nurse \\
\hline 24 & The documentation device has a touch screen & SHOULD & nurse \\
\hline
\end{tabular}

quality of the connection, and the usage of vital sign measurement. After each consultation, the level of usability and acceptance from the GP was evaluated by a questionnaire. Further questionnaires were applied to determine the type and number of technical problems concerning the audio or video connection. In structured Interviews, a subset of patients was asked about their emotion regarding the telemedicine system compared to the current situation. Finally, nurses and nursing management were briefly interviewed on their acceptance in a standardized manner.

\section{Results}

The TeleDoc-system was designed by an interdisciplinary scientific team including a GP, nurses, and elderly patients, as well as engineers and software developers.

\section{Hardware Construction}

Used as prototype 1, a medically licensed wheeled rack (CIM med GmbH, Munich, Germany) was equipped by the Docs in Clouds TeleCare GmbH Aachen, Germany, with a pivoted Power over Ethernet camera including 28times zoom (SNC-EP550 Network PTZ Camera, Sony Corporation, Tokyo, Japan), a medical keyboard, and a medical panel computer (WMP-153, Wincomm, Taiwan) that could be controlled with a touch function (Figure 3). Audio communication was enabled via a Digital Enhanced Cordless Telecommunication (DECT) headset. The power supply was provided via multiple sockets and a power supply box at the foot of the wheeled stand. This box also contained the battery and the power injector for the Power over Ethernet camera. An internet connection was established using a mobile broadband router with two integrated subscriber identity module (SIM) cards.

Since not only video-telephony was pointed out to be crucial but also point-of-care measurements such as ECGbased rhythm analysis, blood pressure, oxygen saturation, heart and respiration rate, various hardware components and medical devices were integrated. 


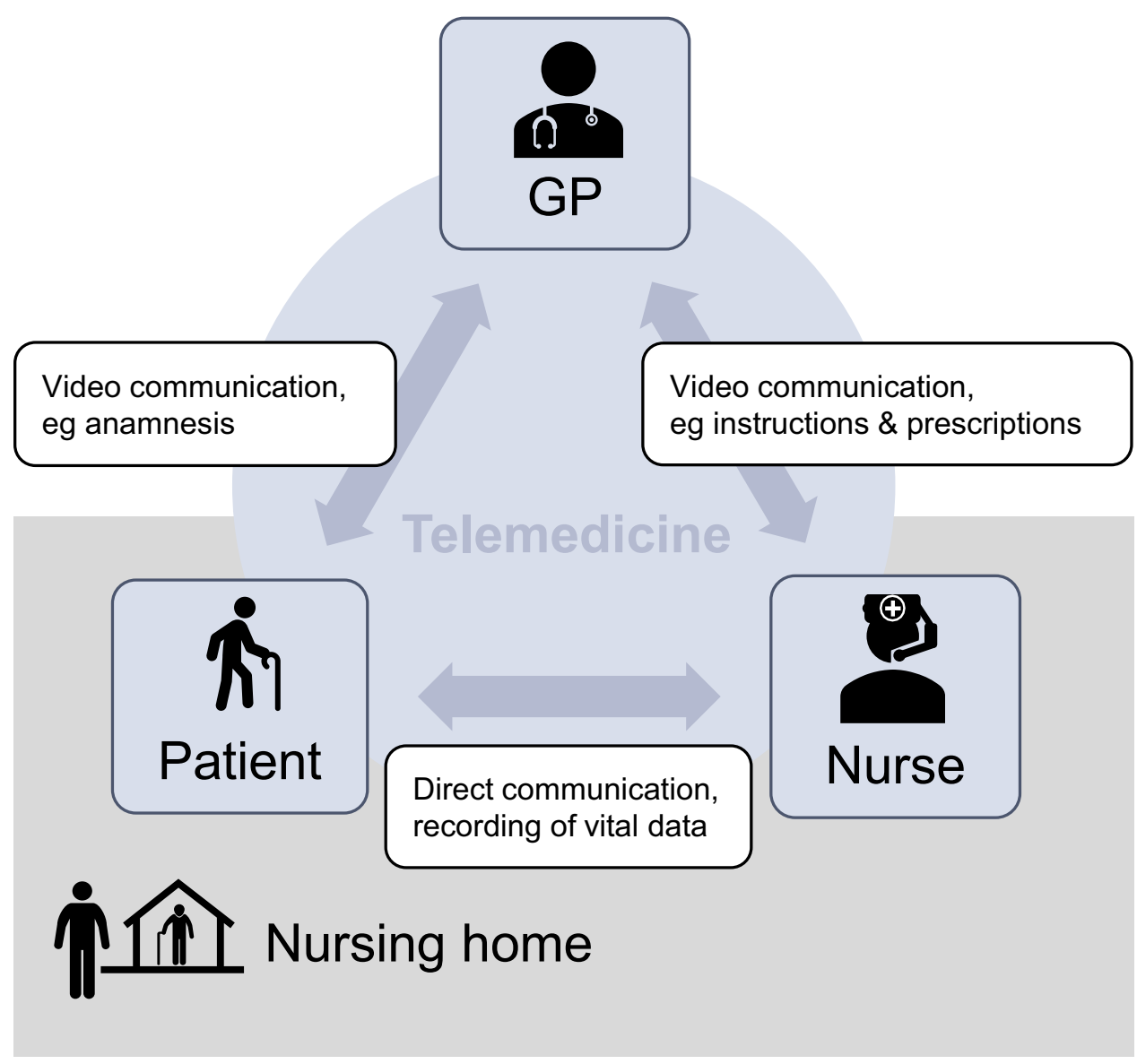

Figure 2 Teleconsultation contributes to an improved communication chain. Whereas telecommunication between general practitioner (GP) and patient is mainly necessary to build trust, the purpose of telecommunication between GP and nurse is to convey therapy instructions.

For vital sign measurement, a patient monitor (Philips MP2, Philips GmbH, Hamburg, Germany) was integrated to enable the recording of heart frequency, oxygen saturation, and blood pressure. Furthermore, a 4-channel or even 12channel-ECG was available for cardiac diagnosis, which could be telemedically transferred to the GP. A basket for monitor cables and additional equipment was also provided.

The GP could make teleconsultations using his own computer with regular equipment (mouse and keyboard) complemented by two monitors and a headset. After the installation of the required software, the GP's computer was connected to the chosen virtual private network (VPN) and received all data from the wheeled stand in the nursing home connected to the same VPN server by the mobile broadband router. The VPN network was coordinated by a VPN concentrator (LANCOM vRouter, LANCOM Systems GmbH, Aachen, Germany), which was also connected to a private cloud. Within the private cloud, a TeleDoc Server managed the communication, authentication, synchronization, and persistence of data.
A video communication server was further included to convey the audio/video connection, as well as a Traversal Using Relays around NAT (Network Address Translation) (TURN) server for the transmission of video data in case the firewall prohibited a direct peer-to-peer connection between the GP's and nurse's computers.

After the first field test (Phase 1), a comprehensive evaluation took place. Many patients complained that they could not see the GP themselves during the consultation. Thus, a second display (Portable Monitor 15.6-inch 1920x1080 Full HD, Kenowa Industries, Holland, MI, USA) was installed on the back side of the wheeled stand, which was directed to the patient on which the camera picture of the practitioner was displayed. Moreover, the use of a headset was evaluated as impracticable for the nurse and patient because both wanted to be continuously part of the conversation with the GP. Accordingly, speakers with speakerphone mode (Jabra Speak 710, GN Audio Germany GmbH, Raubling, Germany) were installed, which was suitable for hearing-impaired users. 


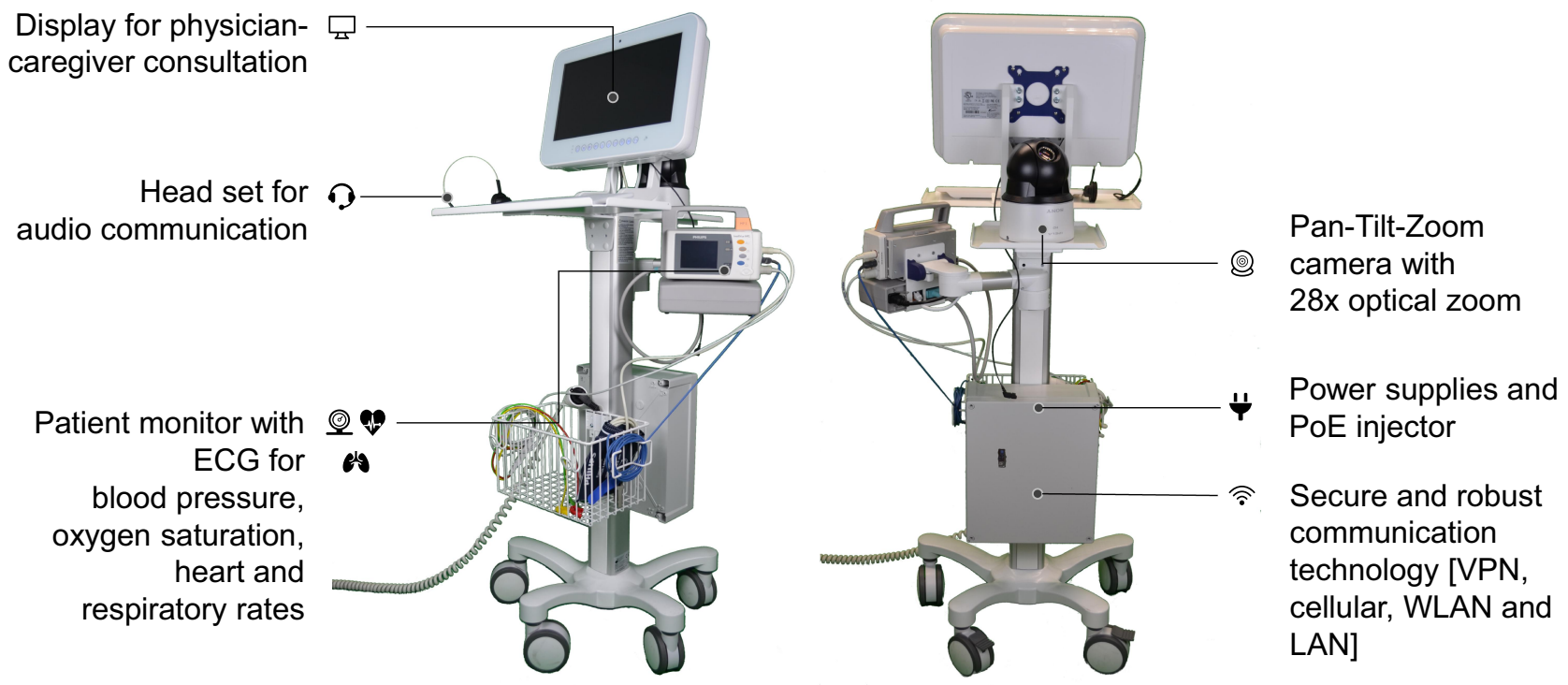

Figure 3 The TeleDoc version I prototype consist of two sides. The front side equips software and hardware components required by the nurse. A Pan-Tilt-Zoom camera is provided at the back side and is directed towards the patient.

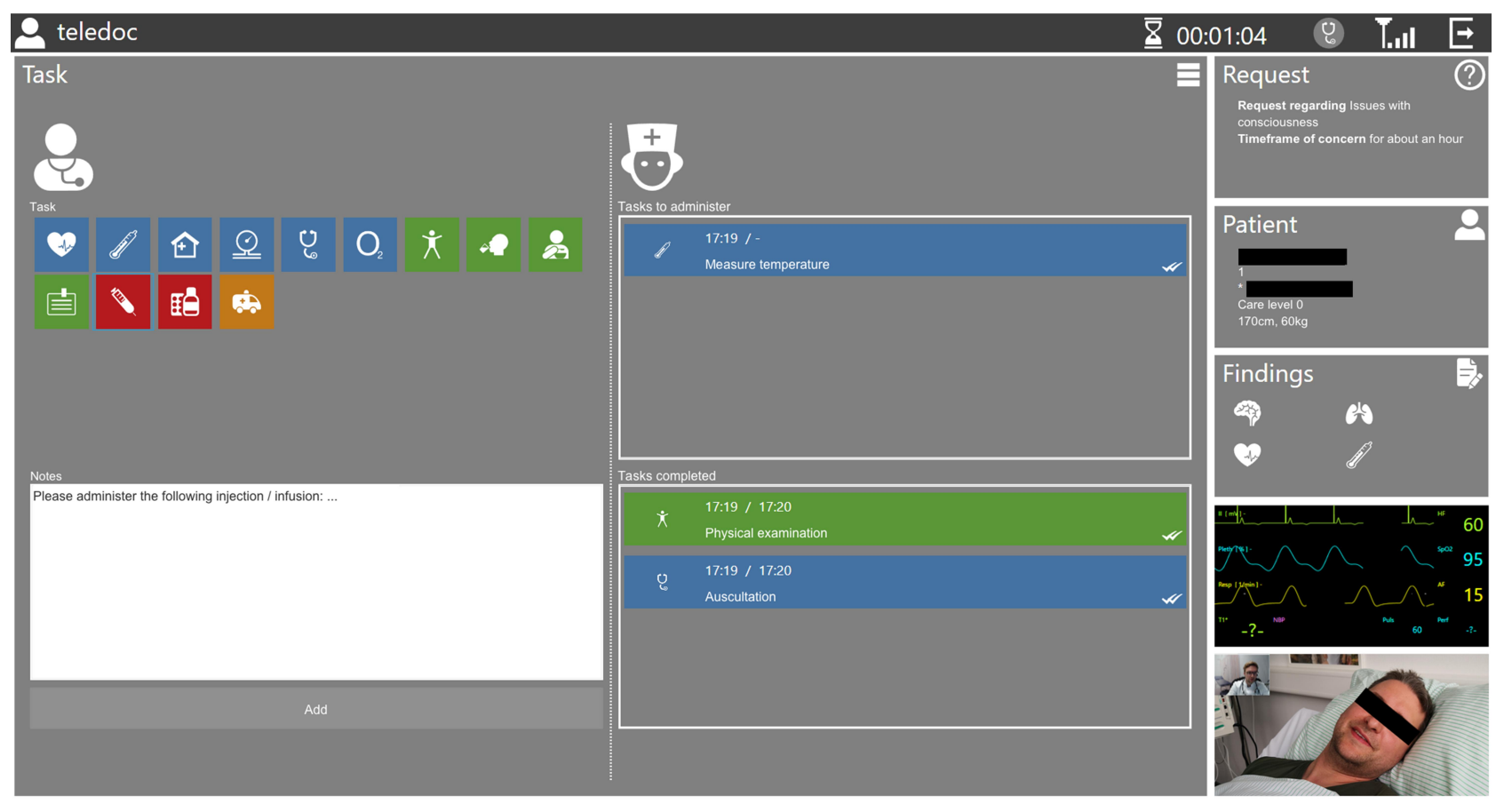

Figure 4 Interface as seen by general practitioner. Panels containing important information on patient data, diagnostic findings, real-time vitals monitoring. Additional panel for real-time patient video image. Colored icons enable quick and easy conveyance of instructions to the nurse.

\section{Software Development}

The TeleDoc System consisted of two users (GP and nurse) with the same code basis at different frontends. Both systems were connected via one server, which ensured the synchronization of all data in real time.
The software was based on an identical core code in which all features were categorized on individual panels (Figure 4). To keep an overview, the display could be customized showing more or less information. Before the consultation, the nurse could provide information on the 
occasion of consultation and on the patient's symptoms. During the consultation, the GP and nurse could utilize panels for patient interaction, instructions, diagnosis, and video following a defined scheme. To prevent errors with documentation, transferred packages needed to be confirmed by a checksum on the application layer. Accordingly, the package was checked by the server after synchronization for all users. Those two steps of verification were depicted by one or two hooks (similar to the WhatsApp icon for sent and received messages).

Via a connector between the nurse's computer and the patient's monitor, all information on the patient's vital signs could be transformed into small packages suitable for streaming and displayed in real time on both the GP's and the nurse's interface.

\section{Field Test}

In the first phase of the field test, a total of 19 patients were consulted. After the first adaptation phase resulting in prototype version 2, another 37 consultations took place (19 in Phase $2 \mathrm{a}$ and 18 in phase $2 \mathrm{~b}$ ). Concerning all consultations, 55 were routine, and one was due to an emergency. Eighteen consultations were interrupted by connection problems, most of them in phase 1, which could be resumed after rebooting the system. In that context, fluctuating connection speeds of the mobile network were registered. By adding the ability to the video connection module to switch the resolution and the compression of the video connection during the live streaming, disconnections hardly reoccurred after the adaptation phase 2 .

During all 55 consultations, audio-visual communication with the patient took place. The patient monitor for vital sign measurement was used in more than half of the consultations (33 cases of blood pressure measurement, 29 cases of electrocardiogram recording, and 26 cases of oxygen saturation and pulse measurement). In seven consultations the administration of medication was instructed by the GP. In the emergency consultation, comprehensive vital sign diagnostics took place, followed by hospitalization.

The duration of consultations was measured using the connection times. There was a declining tendency throughout the field test. Within the first phase, sole audio/video consultation of the GP (without the usage of the patient's monitor) lasted 5:57 minutes on average (median: 5:51 minutes). In phase 2, the average time started at 6:14 (median: 6:28 minutes) minutes and decreased to below five minutes (average 4:27 minutes, median: 4:06 minutes) within the last three months. In cases of vital sign measurement, the consultation took ten minutes longer at the beginning of the field test $115: 47$ minutes, median: 13:43 in phase 1). Subsequently, the average connection time decreased to 13:15 (median: 9:36) minutes in phase $2 \mathrm{a}$ and 11:24 (median: 11:22) minutes in phase $2 \mathrm{~b}$ (Figure 5).

Using questionnaires, usability and acceptance from the GP were measured $(n=45)$. In 96\% (43/45) cases, the teleconsultation was evaluated as "not time-consuming".

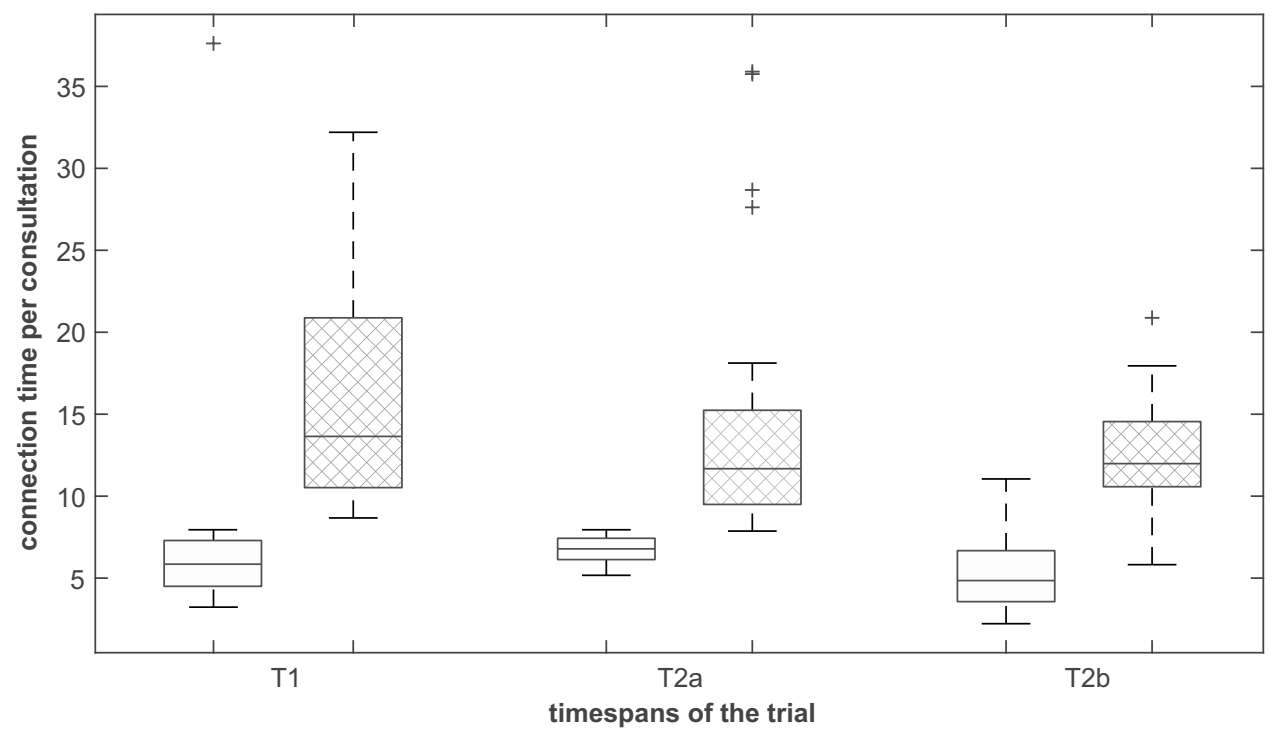

Figure 5 Comparison of physician's connection times a) using solely audio-video connection (white boxes) and b) using devices for vital data measurement (shaded boxes) in the individual timespans of the trial $(\mathrm{I}, 2 \mathrm{a}$ and $2 \mathrm{~b})$. 
Table 2 Benefits of Teleconsultations for Patient, Nurse, Nursing Management and General Practitioner

\begin{tabular}{|c|c|}
\hline Role & Benefits \\
\hline Patient & $\begin{array}{l}\text { - Quick and uncomplicated access to the general } \\
\text { - } \text { Practitioner } \\
\text { - Fewer hospitalizations } \\
\text { - } \text { - Imprentia } \\
\text { - Improvement of care quality }\end{array}$ \\
\hline Nurse & $\begin{array}{l}\text { - Extension of medical abilities } \\
\text { - Possibility of consulting the doctor } \\
\circ \text { immediately, especially at night in emergency } \\
\text { situations } \\
\circ \text { easily in unclear situations } \\
\text { - No need to physically transport the patient to } \\
\text { the doctor }\end{array}$ \\
\hline $\begin{array}{l}\text { Nursing } \\
\text { Management }\end{array}$ & $\begin{array}{l}\text { - Quality improvement of medical care } \\
\text { - Legal certainty for staff } \\
\text { - Unique characteristic towards competing care } \\
\text { - facilities } \\
\text { istrative costs and preventing loss of revenue } \\
\text { due to patient absence }\end{array}$ \\
\hline $\begin{array}{l}\text { General } \\
\text { Practitioner }\end{array}$ & $\begin{array}{l}\text { - More frequent consultations } \\
\text { - Time saving compared with regular home visits } \\
\text { - Reduced travel time to patient }\end{array}$ \\
\hline
\end{tabular}

Simultaneously, the patient's state of health could be assessed well in 98\% (44/45) of all cases, and therefore, no home visit was necessary in $96 \%(43 / 45)$ of all consultations. Telemedical diagnosis and therapy were rated as "easily feasible" in $98 \%(44 / 45)$ of all cases.

Most notably because of the involvement of the elderly people themselves, their feedback was markedly positive. In the structured, all patients confirmed that the doctor's attendance rate is low and they looking forward to see the doctor "though the television" more often especially when they were seriously ill. During the period of the study the caregivers could ask questions to the doctor about residents which seem to develop unclear symptoms once a week. According to the GP, this possibility reduced the count of GP-Emergency visits to the retirement homes on weekends from at least one or more to zero. In addition to that, various benefits were mentioned by the involved GP, the nursing management, and the nurses which also corroborate the acceptance of the system (Table 2).

\section{Discussion}

Through an inter-professional requirement analysis and the inclusion of elderly patients in the development process, it was possible to implement a telemedical system connecting a nursing home with a GP. First field tests revealed special requirements for elderly patients including an extra-large monitor displaying the GP, as well as speakers to make communication possible even with age-related deafness. The integrated medical devices have turned out to be helpful and even necessary for careful and reliable decision making by the GP. Involved patients, nurses, and the general practitioner itemize various specific benefits including economic, personal, and altruistic issues.

In contrast to existing video-telephony services, the TeleDoc system consists of various hardware components and integrated medical devices. One of the major aims was to rate their practical and clinical value. Although ECG is rarely used during usual home visits, it was explicitly requested by the GP to support decision making. During the field tests, ECG and vital data assessment (pulse, blood pressure, and oxygen saturation) was requested in up to $59 \%$ of performed teleconsultations. Different senses, for example, measuring the pulse regarding force and rhythm, require manual touching. Thus, for adequate medical diagnosis from afar, onsite medical assistance and appropriate technical equipment are required.

The need for additional electronic equipment (eg, a digital stethoscope) was voted "valuable" and applied in previous studies. ${ }^{19,20}$ Furthermore, the report of ambulatory care sensitive conditions in Germany shows that various hospitalizations could be easily detected by additional devices and therefore also be prevented. ${ }^{18}$

In addition to the hardware concept, software development was also iteratively performed involving all relevant stakeholders. The most significant software features include a safe and reliable transmission and documentation of medical findings and instructions to ensure legal certainty for both doctor and nurse. Regarding non-functional requirements, ease of use and giving a good overview were implemented as requested by the users. By doing so, the required time for performing a teleconsultation including documentation was optimized. The teleconsultation was considered "not time-consuming" in 96\% (43/45) of the cases. Medical decision-making including diagnosis and therapy were rated as "easily feasible" in $98 \%$ (44/45) of all cases.

In a study examining teleconsultations with geriatric nursing homes, Sävenstedt et al considered an adequate 
application of telemedicine useful in numerous inquiries, leading to various actions such as dosage adjustment of medications. ${ }^{20}$ In general, patients were satisfied and openminded towards this method. ${ }^{21,22}$

Whereas teleconsultations required 5:57 minutes on average in the first study phase, the time increased to 6:14 minutes within phase $2 a$, and finally decreased to 4:27 minutes within the last three months. As a matter of fact, the required time was longer when vitals or ECG were assessed. The total time consumption on average was $15: 47,13: 15$ and 11:24 minutes in study phase $1,2 \mathrm{a}$, and $2 b$. In phase $2 a$ the median departed a little bit $(9: 36)$ from the average but further aligns with the average in $2 b$. Comparing the average with the median shows a longer adaption time for the teleconsultation with measurements. Nevertheless, effective time in average or as median requirement is alike usual visits at the medical practice. $^{23,24}$

Whereas the decreased time within the last time period indicates a learning curve effect, the initial increase was based on the first major reconstruction. Since the beginning of the second time period, the patient was enabled to talk directly to his GP due to the integration of an additional display and speaker. The additional time required in cases when medical devices were applied dropped from about 10 minutes to 7 minutes. This might also be associated with training effects. Basically, it has been shown that teleconsultation takes up less time of the GP than regular home visits do. Telemedicine enables the GP to pass from one patient to another in almost no time, which saves on costs and is environmentally friendly. However, integration of teleconsultations into the physician's everyday practice requires further considerations and change management.

Most notably because of the involvement of the elderly people, their feedback was markedly positive. Since applications like WhatsApp, Skype and Facetime are already well established in everyday life - eg, to communicate with children and grandchildren - the acceptance to use a similar system to keep contact with their GP was high. Besides, the understanding of the increasing lack of physicians - especially in rural regions - was high: "videotelephony is much better than no contact at all".

Teleconsultations are relatively resource-efficient, a quicker and more uncomplicated access to the GP is enabled. Therefore, "home visits" can be performed more frequently by the GP leading to a closer physician-patient relation and might contribute to an improved medical care quality and patient satisfaction as stated in the structured interview. One of the major benefits pointed out by all stakeholders was "fewer hospitalizations" since numerous hospital admissions might be avoidable.

Several studies pointed out, that almost $40 \%$ of hospitalizations of nursing home residents are unnecessary. ${ }^{11}$ Especially, when typical "ambulatory care sensitive conditions" are present such as "Other diseases" of the circulatory system, hypertension, and diseases of urinary system, estimated hospitalization preventability ranges from $76 \%$ to $86 \%{ }^{18}$

Whereas scheduled (physical) home visits by the GP took place as usual, during the study period no additional (unplanned) home visit was necessary. It was reported that urgent visits of the doctor are usually required almost every weekend. Although not within the scope of our study, anyhow one emergency consultation was performed by chance.

The benefits mentioned by the nursing management include quality improvement of medical care, legal certainty for caregivers, and cost savings especially due to the avoidance of unnecessary hospitalizations. ${ }^{11,18}$

General practitioner care in retirement homes is compounded by the fact that GPs are increasingly missing especially in rural areas - where retirement homes usually are located. In cases of urgent need for medical decisions, GPs are often not available within a reasonable time frame. Therefore, nurses are commonly pressured to make an emergency call with the consequence of subsequent hospital admission. However, a huge number of those "unclear situations" do not require manual medical skills but "only" medical decision making and bearing responsibility. ${ }^{20}$ In those cases, a teleconsultation provides legal certainty for the caregivers and - due to an adequate documentation - also for the doctor.

Cost savings are - on the one hand - caused by less administration efforts that are associated with hospital admissions. On the other hand, daily charges are partly cut when the resident is outside the nursing home (in this case due to hospitalization).

Additionally, the extension of medical abilities and competences was considered "valorizing" which might have positive effects for staff recruitment and satisfaction.

Another issue that the current COVID-19 crisis brought to light is lowering the risk of contagion. Health care workers have an increased risk of catching communicable diseases, including COVID-19, as they are in constant contact with patients. Thus, during the current 

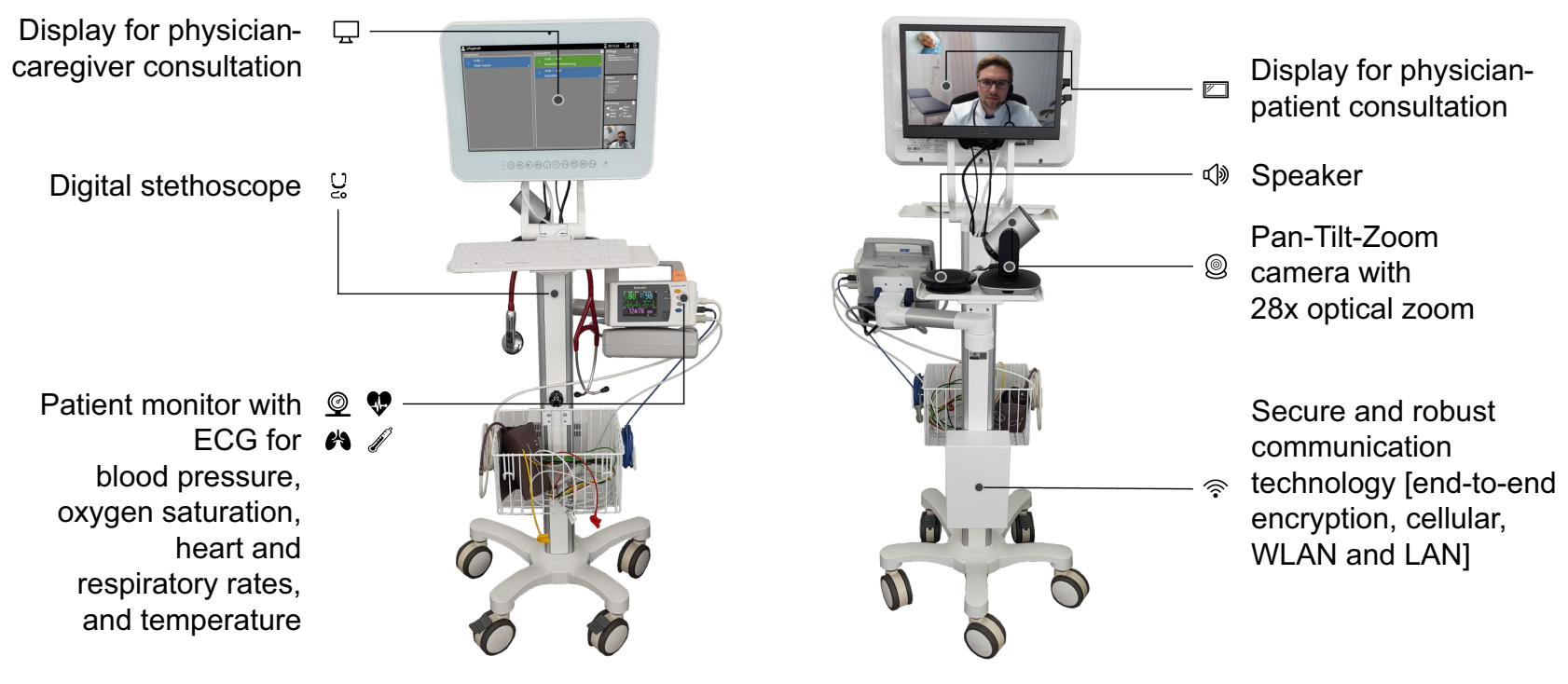

Figure 6 In contrast to prototype version I, the final TeleDoc product provides a patient display, a digital stethoscope, and an external speaker.

circumstances, home visits or visits to the doctor's office are completely discouraged (if they are not mandatory), because they represent an extra risk for this group. Telemedicine is a great solution to assure a continuous medical care and at the same time physical distancing. With the telemedicine system, the general practitioners will have the chance to make their regular home visits, without having second thoughts whether they will potentially or not infect the patients.

Upon the completion of the study, the panel computer takes over the access point functionality. To further improve data safety, end-to-end encryption was added to transportation encryption. As a result, VPN is no longer necessary (Figure 6).

\section{Conclusion}

A holistic telemedical system including integrated medical devices was successfully developed and has turned out to be helpful and even necessary for careful and reliable decision making by the GP. First test results of the telemedical system show high acceptance in retirement homes. Involved patients, nurses, and the general practitioner itemize various specific benefits including economic, personal, and altruistic issues. Another issue that the current COVID-19 crisis brought to light is lowering the risk of contagion. GPs can replace their home visits by using telepresence combined with point of care measures. Further research should be conducted to evaluate patient outcomes and the health economic consequences of telemedicine in nursing homes.

\section{Data Sharing Statement}

The datasets generated during and/or analysed during the current study are available from the corresponding author on reasonable request. The datasets from the questionnaire generated during the current study are available in the Zenodo repository, https://doi.org/10.5281/zenodo. $\underline{3973578 .}$.

\section{Acknowledgments}

We thank all the involved residents (patients), the nursing management and the professional caregivers from the involved retirement home for their active participation. Furthermore, we would also like to show our gratitude to Dr. Rafael Walocha for sharing his time, expertise and knowledge.

\section{Disclosure}

Marian Ohligs and Michael Czaplik are employed by Docs in Clouds $\mathrm{GmbH}$ (Aachen, Germany), providing telemedical services and consulting. Michael Czaplik and Rolf Rossaint report personal fees from and are shareholders of 
Docs in Clouds GmbH, Aachen, Germany. The authors report no other conflicts of interest in this work.

\section{References}

1. Jetty A, Moore MA, Coffman M, Petterson S, Bazemore A. Rural family physicians are twice as likely to use telehealth as urban family physicians. Telemed $J$ E-Health off $J$ Am Telemed Assoc. 2018;24 (4):268-276.

2. Brinks R, Tamayo T, Kowall B, Rathmann W. Prevalence of type 2 diabetes in Germany in 2040: estimates from an epidemiological model. Eur J Epidemiol. 2012;27(10):791-797. doi:10.1007/s10654012-9726-2

3. Yeh RW, Sidney S, Chandra M, Sorel M, Selby JV, Go AS. Population trends in the incidence and outcomes of acute myocardial infarction. N Engl J Med. 2010;362(23):2155-2165. doi:10.1056/ NEJMoa090 8610

4. Nowossadeck E. Population aging and hospitalization for chronic disease in Germany. Dtsch Arzteblatt Int. 2012;109(9):151-157.

5. Bomsdorf E, Babel B, Kahlenberg J. Care need projections for Germany until 2050. In: Doblhammer G, Scholz R, editors. Ageing Care Need Qual Life Perspect Care Givers People Need Care. Wiesbaden: VS Verlag für Sozialwissenschaften; 2010:29-41.

6. Koch K, Miksch A, Schürmann C, Joos S, Sawicki PT. The German health care system in international comparison: the primary care physicians' perspective. Dtsch Arzteblatt Int. 2011;108(15):255-261.

7. Pohontsch NJ, Hansen H, Schäfer I, Scherer M. General practitioners' perception of being a doctor in urban vs. rural regions in Germany - a focus group study. Fam Pract. 2018;35(2):209-215. doi:10.1093/fampra/cmx083

8. Steinhaeuser J, Joos S, Szecsenyi J, Miksch A. A comparison of the workload of rural and urban primary care physicians in Germany: analysis of a questionnaire survey. BMC Fam Pract. 2011;12:112. doi:10.1186/1471-2296-12-112

9. Fassier T, Duclos A, Abbas-Chorfa F, et al. Elderly patients hospitalized in the ICU in France: a population-based study using secondary data from the national hospital discharge database. J Eval Clin Pract. 2016;22(3):378-386. doi:10.1111/jep.12497

10. Burgdorf F, Sundmacher L. Potentially avoidable hospital admissions in Germany: an analysis of factors influencing rates of ambulatory care sensitive hospitalizations. Dtsch Arzteblatt Int. 2014;111(13):215-223.

11. Ouslander JG, Berenson RA. Reducing unnecessary hospitalizations of nursing home residents. $N$ Engl J Med. 2011;365(13):1165-1167. doi:10.1056/NEJMp1105449
12. Marcantonio ER. Delirium in hospitalized older adults. $N$ Engl J Med. 2017;377(15):1456-1466. doi:10.1056/NEJMcp1605501

13. Bergrath S, Reich A, Rossaint R, et al. Feasibility of prehospital teleconsultation in acute stroke - a pilot study in clinical routine. PLoS One. 2012;7(5):e36796. doi:10.1371/journal.pone.0036796

14. Brokmann JC, Conrad C, Rossaint R, et al. Treatment of acute coronary syndrome by telemedically supported paramedics compared with physician-based treatment: a prospective, interventional, multicenter trial. J Med Internet Res. 2016;18(12):e314. doi:10.2196/jmir.6358

15. Czaplik M, Bergrath S, Rossaint R, et al. Employment of telemedicine in emergency medicine. Clinical requirement analysis, system development and first test results. Methods Inf Med. 2014;53 (2):99-107. doi:10.3414/ME13-01-0022

16. van Gelder VA, Scherpbier-de Haan ND, van Berkel S, et al. Webbased consultation between general practitioners and nephrologists: a cluster randomized controlled trial. Fam Pract. 2017;34 (4):430-436. doi:10.1093/fampra/cmw131

17. Iversen MM, Espehaug B, Hausken MF, et al. Telemedicine versus standard follow-up care for diabetes-related foot ulcers: protocol for a cluster randomized controlled noninferiority trial (DiaFOTo). JMIR Res Protoc. 2016;5(3):e148. doi:10.2196/resprot.5646

18. Fischbach D. Krankenhauskosten ambulant-sensitiver Krankenhausfälle in Deutschland [Hospital Costs of Ambulatory Care-Sensitive Conditions in Germany]. Gesundheitswesen. 2016;78(03):168-174. doi:10.1055/s-0035-1548779

19. Esterle L, Mathieu-Fritz A. Teleconsultation in geriatrics: impact on professional practice. Int J Med Inf. 2013;82(8):684-695. doi:10.1016/j.ijmedinf.2013.04.006

20. Sävenstedt S, Bucht G, Norberg L, Sandman PO. Nurse-doctor interaction in teleconsultations between a hospital and a geriatric nursing home. J Telemed Telecare. 2002;8(1):11-18. doi:10.1258/1357633 021937406

21. Chae YM, Heon Lee J, Hee HS, Ja Kim H, Hong Jun K, Uk Won J. Patient satisfaction with telemedicine in home health services for the elderly. Int $J$ Med Inf. 2001;61(2):167-173. doi:10.1016/S13865056(01)00139-3

22. Jaatinen PT, Aarnio P, Remes J, Hannukainen J, Köymäri-Seilonen T. Teleconsultation as a replacement for referral to an outpatient clinic. $J$ Telemed Telecare. 2002;8(2):102-106. doi:10.1258/135763302 1937550

23. Carr-Hill R, Jenkins-Clarke S, Dixon P, Pringle M. Do minutes count? Consultation lengths in general practice. J Health Serv Res Policy. 1998;3(4):207-213. doi:10.1177/135581969800300405

24. Cape J. Consultation length, patient-estimated consultation length, and satisfaction with the consultation. Br J Gen Pract. 2002;3.
Clinical Interventions in Aging

\section{Publish your work in this journal}

Clinical Interventions in Aging is an international, peer-reviewed journal focusing on evidence-based reports on the value or lack thereof of treatments intended to prevent or delay the onset of maladaptive correlates of aging in human beings. This journal is indexed on PubMed Central, MedLine, CAS, Scopus and the Elsevier
Bibliographic databases. The manuscript management system is completely online and includes a very quick and fair peer-review system, which is all easy to use. Visit http://www.dovepress.com/ testimonials.php to read real quotes from published authors. 